\title{
Differential Diagnosis of Amniotic Band and Intrauterine Synechiae in Second Trimester Pregnancy: Case Report
}

\author{
Bekir KAHVECI ${ }^{1}$, Yasemin DOGAN', Gaye KAHVECI², Muhammet Hanifi BADEMKIRAN' \\ Diyarbakir, Turkey
}

\begin{abstract}
Amniotic band sequence describes highly variable spectrum of congenital anomalies that occur in association with amniotic bands. Intrauterine synechiae is a condition in which scar tissue develops within the uterine cavity. We aim to present a case of amniotic band sequence and a case of intrauterine synechiae and compare their ultrasonographic findings. Ultrasonography revealed contraction ring in the right groin area of the first fetus but second fetus was healthy. The diagnosis of amniotic band sequence is based upon the presence of characteristic structural findings on prenatal ultrasound or postnatal physical examination. The diagnosis of intrauterine adhesions is based upon visualization of intrauterine adhesions either directly by hysteroscopy or indirectly by other imaging studies. The floor of the intrauterine adhesions is widely and lateral of the uterine cavity and occurs from four layers while the amniotic band is a thin layer and forms fluctuations in the amniotic fluid. Amniotic bands may cause variable spectrum of congenital anomalies whereas intrauterine adhesions do not increase the probability of fetal anomaly because it is out of the amniotic cavity.
\end{abstract}

Keywords: Amniotic band sequence, Intrauterine adhesions, Differential diagnosis

Gynecol Obstet Reprod Med 2020;26(3):229-231

\section{Introduction}

Amniotic band sequence describes highly variable spectrum of congenital anomalies that occur in association with amniotic bands (1). The estimated incidence of ABS ranges from $1: 1200$ to $1: 15000$ in live births (2). It is called a sequence because the pattern of congenital anomalies results from a single defect that can be produced by a variety of different etiologies. The pathogenesis of both amniotic bands and ABS are not firmly established. Etiology and pathogenesis are

\footnotetext{
${ }^{1}$ Diyarbakır Gazi Yaşargil Training and Research Hospital Department of Obstetrics and Gynecology, Diyarbakir, Turkey

2 Dicle University, Department of Family Medicine, Diyarbakır, Turkey Address of Correspondence: Bekir Kahveci

Health Sciences University Gazi Yasargil Research and Training Hospital

Department of Obstetrics and Gynecology 21010 Diyarbakır, Turkey drbekirkahveci@hotmail.com

Submitted for Publication: $\quad$ 17. 07. 2018

Accepted for Publication: $\quad$ 20. 12.2018

ORCID IDs of the authors: $\quad$ BK: 0000-0002-8729-1669

YS:0000-0002-7492-0470, GK:0000-0002-2614-4411 MHB: 0000-0002-9350-582X
}

\begin{tabular}{c|c}
\hline Quick Response Code: & Access this article online \\
\cline { 2 - 2 } & Website: www.gorm.com.tr \\
& e- mail: info@gorm.com.tr \\
\hline
\end{tabular}

How to cite this article: Kahveci B. Dogan Y. Kahveci G. Bademkıran MH. Differential Diagnosis of Amniotic Band and Intrauterine Synechiae in Second Trimester Pregnancy: Case Report. Gynecol Obstet Reprod Med. 2020;26(3):229-231 heterogeneous and controversial. Loose strands of amnion are believed to adhere and then entangle the embryo/fetus or germ disc, resulting in mechanical or vascular disruption of developing structures (3). Clinical manifestations include limb and digital amputation, constriction rings, craniofacial abnormalities, body wall defects, syndactyly, auto transplanted tissue on skin tags, clubfoot, hand deformities, spinal defects, and lung hypoplasia. Abdominal or thoracic contents may herniate through a body wall defect and into the amniotic cavity (4).

Intrauterine adhesions, or intrauterine synechiae, is a condition in which scar tissue develops within the uterine cavity. Intrauterine adhesions accompanied by symptoms (e.g., infertility, amenorrhea) are also referred to as Asherman syndrome (5). The true prevalence of intrauterine adhesions is difficult to establish, because the condition is rare in the general population and often asymptomatic. Estimates of the prevalence range from $1.5 \%$ as an incidental finding at hysterosalpingogram to $21.5 \%$ of women with a history of postpartum uterine curettage (6). Intrauterine adhesions typically result from intrauterine trauma associated with a surgical procedure, although infection may play a minor contributing role. Severe intrauterine adhesive disease is primarily caused by curettage for pregnancy complications, such as missed or incomplete abortion or postpartum hemorrhage (7). The role of postpartum or post abortion infection in adhesion formation is controversial, and data are limited. Adhesions can also develop in the non-gravid uterus as a result of endometrial injury from procedures such as myomectomy or curettage for indications not related to pregnancy (8). Curettage of the endometrium 
can result in trauma to the basalis layer. The basalis layer of the endometrium appears to be most susceptible to damage in the first four postpartum or post-abortion weeks. Clinical manifestations of severe intrauterine adhesive disease include infertility, recurrent pregnancy loss, menstrual irregularities (amenorrhea, hypomenorrhea), and cyclic pelvic pain.

\section{Case Report}

Two patients who were referred to our clinic for anatomic screening were evaluated. The first pregnant woman aged 25 years presented during her $22^{\text {nd }}$ week of gestation for a routine fetal anatomic scan. Her medical history was not significant for any clinical condition. The fetus had a normal anatomic scan but fetus had amniotic band sequence. This amniotic band was thin and there was fluctuations in amniotic fluid. These findings are clearly visible in the first image (Figure 1a). Evaluation with 3D ultrasonography in the antenatal period revealed a contraction ring in the right groin area of the fetus (Figure 2).

Second pregnant woman aged 30 years presented during her $21^{\text {st }}$ week of gestation for a routine fetal anatomic scan. Her medical history included one curettage for pregnancy complication. The fetus had a normal anatomic scan but she had intrauterine synechiae. A healthy baby was born at the 38th gestational week. The floor of the intrauterine synechiae was widely and lateral of the uterine cavity. These findings are clearly visible in the right image of Figure $1 \mathrm{~b}$.

The first fetus was evaluated physically after birth. We observed contraction scar in right pubic area on physical examination (Figure 3 ). We informed the family about this condition.

Informed consent has been obtained from both patients.

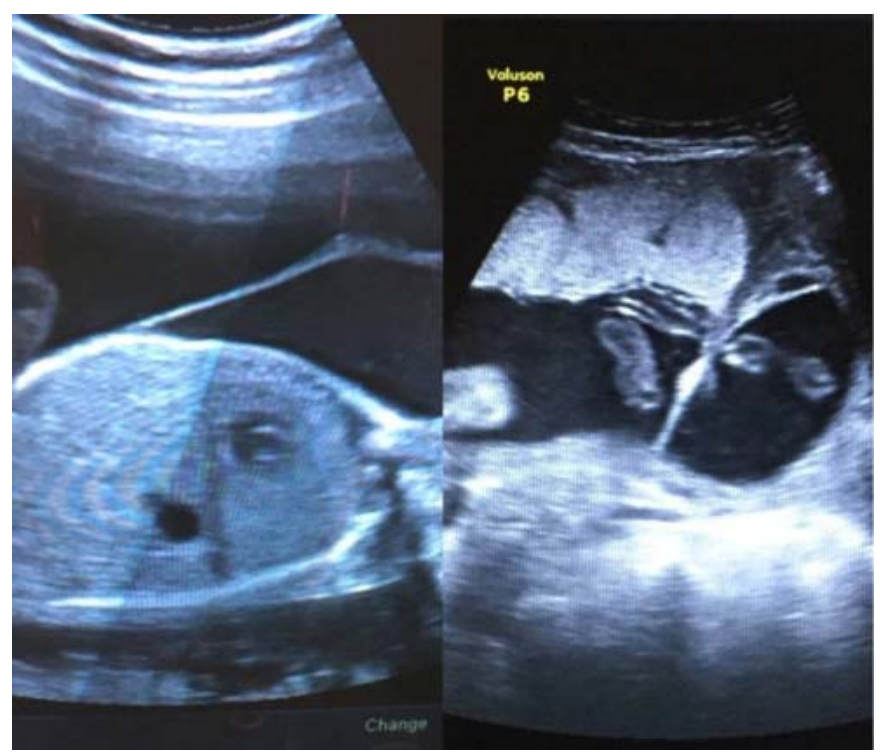

Figure 1a: The first image is amniotic band and 1b: Second image is intrauterine synechiae.Figure 1a: The first image is amniotic band and 1b: Second image is intrauterine synechiae

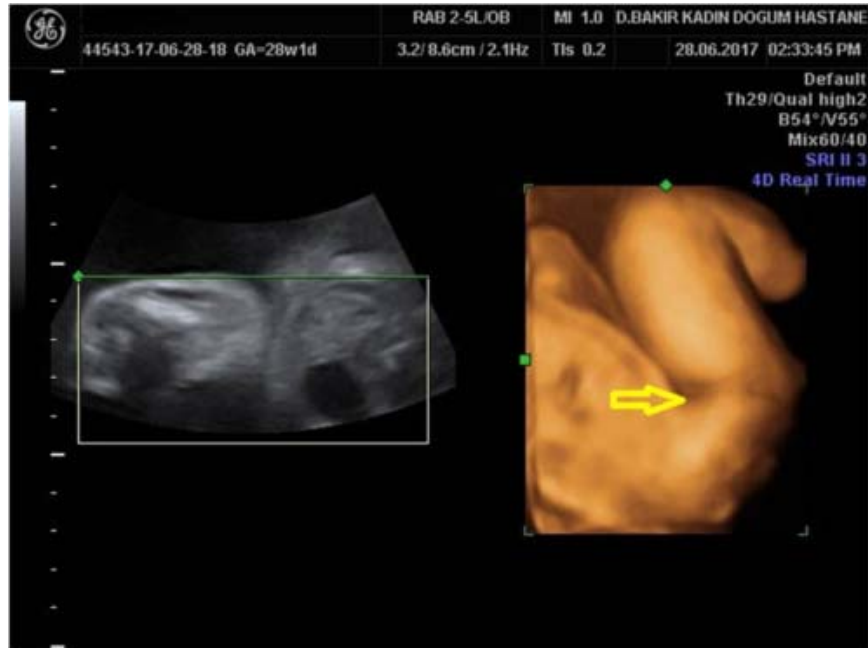

Figure 2: Appearance of the three- $D$, contraction rings in the right groin area

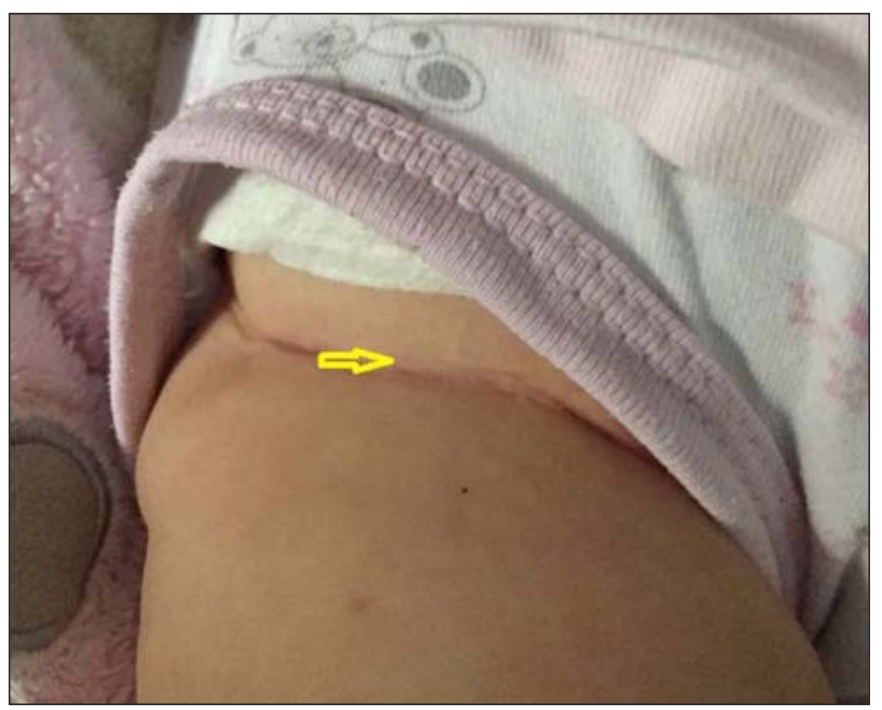

Figure 3: Scarring appearance of the contraction ring in the postnatal period.

\section{Discussion}

The diagnosis of ABS is based upon the presence of characteristic structural findings on prenatal ultrasound or postnatal physical examination of the affected individual. The diagnosis should be suspected when limb amputations or atypical body wall or craniofacial defects are present, or when bands of amnion are seen crossing the gestational sac and adherent to the fetus, restricting its movement. The diagnosis of ABS should not be based on visualization of amniotic abnormalities alone in the absence of fetal structural abnormalities or restricted movement. Antenatal magnetic resonance imaging may be helpful in confirming the diagnosis (7). In our case the fetus had a contraction ring in the right groin area which was confirmed after birth.

Amniotic sheets typically arise when chorioamniotic membranes wrap around uterine synechiae (intrauterine adhesions), thus they have two layers of amnion and two layers of 
chorion (8). They commonly have a widened base along the uterine wall, and may extend to the contralateral uterine wall.

The prenatal and intrapartum management of suspected ABS depends on the type and extent of malformations. All patients should receive information about the fetal abnormalities that have been detected, the possibility of additional undetected abnormalities, prognosis, and the option of pregnancy termination. If the diagnosis of ABS is uncertain, genetic studies are often indicated (7).

The diagnosis of intrauterine adhesions is based upon visualization of intrauterine adhesions either directly by hysteroscopy or indirectly by other imaging studies. The main components of the diagnostic evaluation for intrauterine adhesions are the medical history and hysteroscopy. Pelvic imaging findings may be the initial test that suggests that adhesions are present. In addition, a history of uterine procedures, particularly curettage related to pregnancy, and pelvic infections (endometritis, pelvic inflammatory disease) should be elicited. Saline infusion sonohysterogram (SIS) or hysterosalpingogram (HSG) play a limited role in the diagnosis of intrauterine adhesions. Pregnancies in women with a history of intrauterine adhesions should be considered high risk and may require a consultation from a maternal fetal medicine specialist. Pregnancy complications can include intrauterine growth restriction, preterm delivery, and abnormal placentation (7). The amniotic membranes may be encountered in the uterus with a thin interface that surrounds a uterine synechiae all around. This uterine malformation is called the amniotic leaf and there is no increase in the probability of fetal anomaly because it is out of the amniotic cavity. Likewise, the baby was born healthy in our case with intrauterine adhesion.

Synechiae are intrauterine adhesions that develop from the endometrial lining after an endometrial injury (9). Amniotic sheets form when an existing synechiae comes in contact with the expanding fetal membranes of the chorion and amnion. These membranes then fold around the synechiae, creating a linear four-layered structure comprising two outer amnion layers sandwiching two inner chorion layers, with a variable amount of stretched endometrium or scar tissue (10). As a result of their wrapping around existing uterine synechiae, amniotic sheets always arise from the uterine wall and span from myometrium to myometrium. The fetus is exposed to only the smooth surface of the amniotic membrane; this is in stark contrast to cases of amniotic bands, in which the fetus is exposed to the extraembryonic coelomic space (11). Although the appearance of synechiae changes little during pregnancy, these adhesions may be difficult to identify later during the pregnancy or at delivery probably owing to rupture or displacement during fetal growth and uterine expansion (12). Rarely, synechiae are compressive and compartmentalize the uterus owing to the scar and associated membranes extending tightly across the uterus.
As a result, the floor of the amniotic leaves (intrauterine adhesions) is widely and occurs from four layers (two amniotic and two chorionic) while the amniotic band is a thin layer and forms fluctuations in the amniotic fluid. Amniotic bands may cause variable spectrum of congenital anomalies whereas intrauterine adhesions do not increase the probability of fetal anomaly because it is out of the amniotic cavity. It is important to make a differential diagnosis of these two conditions.

2. This study was presented as an oral presentation by Bekir Kahveci at the $12^{\text {th }}$ International XII. Turkish-German Gynecology Congress in Turkish Republic of Northern Cyprus. Conflict of Interest: No conflict of interest was declared by the authors.

\section{References}

1. Eichhorn MG, Iacobucci JJ, Turfe Z. An unusual craniofacial cleft: amniotic band syndrome as a possible cause. Int J Pediatr Otorhinolaryngol 2015;79(4):616-9.

2. López-Muñoz E, Becerra-Solano LE. An update on amniotic bands sequence. Arch Argent Pediatr 2018;116(3): 409-12.

3. Kostadinov S, Shapiro S. Unusual Case of Amniotic Band Sequence. Int J Surg Pathol 2017;25(5): 430.

4. Deans R, Abbott J. Review of Intrauterine adhesions. J Minim Invasive Gynecol 2010;17(5):555-69.

5. Al-Inany H. Intrauterine adhesions. An update. Acta Obstet Gynecol Scand 2001;80(11):986-93.

6. Gün İ, Muhçu M, Müngen E, Kiliç S, Atay V. Effect of an Amniotic Sheet on Pregnancy Outcomes. J Ultrasound Med 2013;32(5):807-13.

7. Neuman J, Calvo-Garcia MA, Kline-Fath BM, Bitters C, Merrow AC, Guimaraes CV, et al. Prenatal imaging of amniotic band sequence: utility and role of fetal MRI as an adjunct to prenatal US. Pediatr Radiol 2012;42(5):544-51.

8. Tuuli MG, Shanks A, Bernhard L, Odibo AO, Macones GA, Cahill A. Uterine synechiae and pregnancy complications. Obstet Gynecol 2012;119(4):810-4.

9. Jensen KK, Oh KY, Kennedy AM, Sohaey R. Intrauterine Linear Echogenicities in the Gravid Uterus: What Radiologists Should Know. Radiographics 2018;38(2): 642-15.

10. Tan KB, Tan TY, Tan JV, Yan YL, Yeo GS. The amniotic sheet: a truly benign condition? Ultrasound Obstet Gynecol 2005;26(6):639-43.

11. Iqbal CW, Derderian SC, Cheng Y, Lee H, Hirose S. Amniotic band syndrome: a single-institutional experience. Fetal Diagn Ther 2015;37(1):1-5.

12. Bibbo C, Little SE, Bsat J, Botka KA, Benson CB, Robinson JN. Chorioamniotic separation found on obstetric ultrasound and perinatal outcome. AJP Rep 2016;6(3): e337-43. 